\title{
Fractional high-harmonic combs by attosecond-precision split-spectrum pulse control
}

\author{
Philipp Raith, Christian Ott, Christopher P. Anderson, Andreas Kaldun, Kristina Meyer, Martin \\ Laux, Yizhu Zhang, and Thomas Pfeifer \\ Max-Planck-Institut für Kernphysik, Saupfercheckweg 1, D-69117 Heidelberg, Germany
}

\begin{abstract}
Few-cycle laser fields enable pulse-shaping control of high-order harmonic generation by time delaying variable broadband spectral sections. We report the experimental generation of fractional (noninteger) high-harmonic combs by the controlled interference of two attosecond pulse trains. Additionally the energy of the high harmonics is strongly tuned with the relative time delay. We quantify the tuning to directly result from the controlled variation of the instantaneous laser frequency at the shaped driver pulse intensity maximum.
\end{abstract}

High-order harmonic generation (HHG) has thus far been controlled by varying the carrier-envelope phase of the driving laser field [1], by the addition of a second driver field at a different frequency, e.g. the second harmonic [2], or by adaptive pulse control [3,4]. Recently, sculpted optical waveforms shorter than a full laser cycle have been produced in a three-channel super-octave optical field synthesizer [5]. Here, we apply a similar continuous-spectrum pulse shaping method to perform comprehensive, coherent control of HHG [6]. We observe energy-tuning capability of the high-order harmonics and the generation of combs of fractional (noninteger) harmonics caused by the controlled spectral interference of two temporally separated attosecond pulse trains. To employ the splitspectrum time-delay control method, suggested for independent control of several attosecond pulse properties [7], we use a single broadband continuous laser spectrum which is divided into two adjustable spectral sections by a split mirror on a translation stage. The piezoelectric-driven split mirror is situated in the Fourier-plane of a Brewster-prism-based pulse shaper in 4-f configuration (Figure 1a) and enables the introduction of time delays between the spectral subfields with a precision below 400 as. High-order harmonics are produced with such shaped pulses in argon at a laser peak intensity of $\sim 0.44 \mathrm{PW} / \mathrm{cm}^{2}$ and a backing pressure of $\sim 60 \mathrm{mbar}$.

Figure 1c shows the measured harmonic spectrum dependent on the time delay between the spectral components for the energy-asymmetric split spectrum depicted in Figure 1b. The high-order harmonics beat with time delay due to an alternating constructive and destructive interference of the driving subfields at the temporal center of the pulse. The energy of the high harmonics varies with time delay both on attosecond (subcycle) and on few tens of femtoseconds (subfields overlap) time scales. We extracted the physical mechanism and origin behind this pronounced energy tunability. It results from an interference-based modulation of the instantaneous frequency $\omega(t)=\dot{\varphi}(t)$ of the shaped driver pulse at the temporal intensity maximum. The harmonic energy tuning further depends

This is an Open Access article distributed under the terms of the Creative Commons Attribution License 2.0, which permits unrestricted use, distribution, and reproduction in any medium, provided the original work is properly cited. 
on the overall chirp of the driving laser field. We calculate the relative change of the instantaneous laser frequency at the intensity maximum for a chirp of $-10 \mathrm{fs}^{2}, 10 \mathrm{fs}^{2}$ and without chirp. Excellent qualitative and quantitative agreement to the measured relative change of the harmonics' energy is found for a chirp of $-10 \mathrm{fs}^{2}$ (see Figure 1c), which we assume to have been present in the experiment, while it could not be directly measured on target in the vacuum chamber.
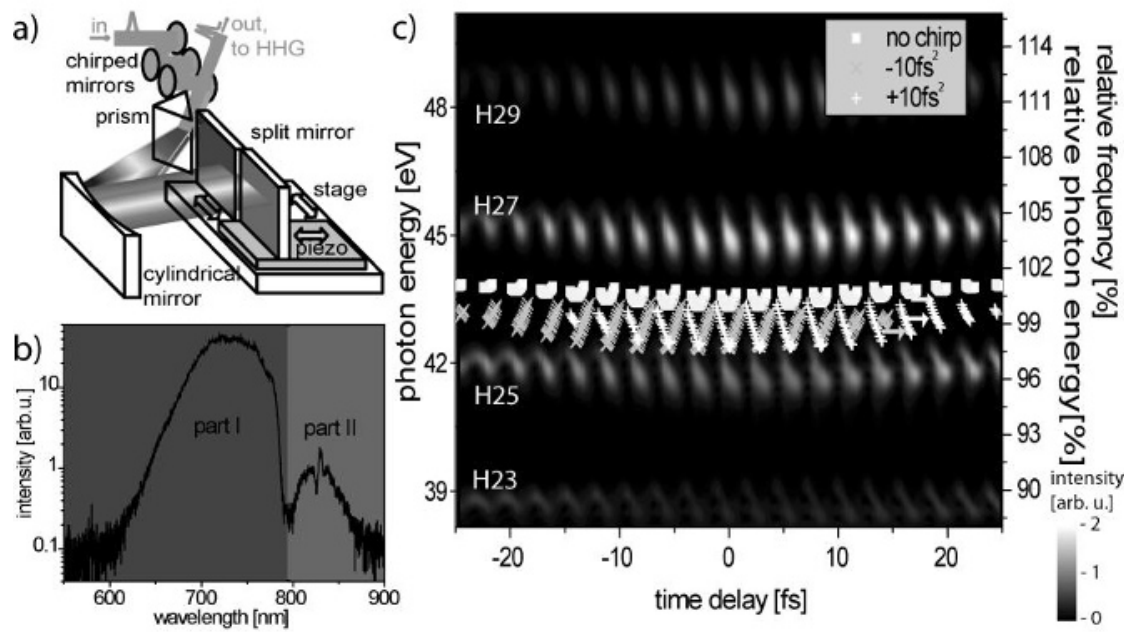

Fig. 1. Energy tuning control of high-order harmonics [6]. a) Setup of the split-spectrum pulse shaper in 4-f configuration. b) Measured continuous two-channel split spectrum with an asymmetric cut (I:II energy ratio $58: 1$ ) set to $795 \mathrm{~nm}$. c) Time-delay dependent energy tunability of measured high-order harmonics and modulation of the instantaneous laser driver frequency at the temporal intensity maximum of the shaped pulse. Excellent quantitative agreement of the relative frequency at the pulse peak (from the model) and measured energy tuning is found considering a chirp of $-10 \mathrm{fs}^{2}$ (crosses), compared to zero chirp (squares) and $+10 \mathrm{fs}^{2}$ (pluses). Note that the relative frequency of $100 \%$ is chosen arbitrary to offset the experimental and model data for better visibility.

For a split spectrum with a more symmetric cut as depicted in Figure 2a we obtain-besides the regular odd harmonics - additional spectral components (Figure 2b) forming fine spectral combs. These combs of fractional (noninteger) harmonics appear at time delays when the temporal beating of the two interfering subfields leads to shaped driving pulses that have two approximately equally intense maxima in time. At each intensity maximum an attosecond pulse train is generated and phase-matched. The spectral interference of these two coherent attosecond pulse trains leads to the combs of noninteger harmonics which have an energy spacing of only $0.3 \mathrm{eV}$, corresponding to the inverse of the temporal separation of the two attosecond pulse trains (Fig 2c). The interference of the attosecond pulses within each pulse train leads to an additional two-omega modulation of the comb as known from the regular odd harmonics.

We compare our experimental results with numerical simulations based on the single-atom response. The electric field of the shaped driving pulse (Figure 2d) is given by the Fourier transform of the measured laser spectrum, where the spectral phase is determined by the cut position and time delay of the two spectral sections. Applying the strong-field approximation for the same laser peak intensity as present in the experiment, we numerically obtain the harmonic spectra, which compare excellently with the experimental data and reproduce all observed spectral effects. This demonstrates the ability of the presented scheme to control high-order harmonic generation on the single atom level. Our simulations give evidence that the split-spectrum control method allows to properly balance the intensity ratio of the two intensity maxima of the shaped laser pulses resulting in two equally intense attosecond pulse trains and a fully modulated spectral comb of fractional harmonics. 
Such combs have full intensity contrast between peak and valleys and could be useful to enhance the spectral resolution in time-resolved spectroscopy or interferometry.
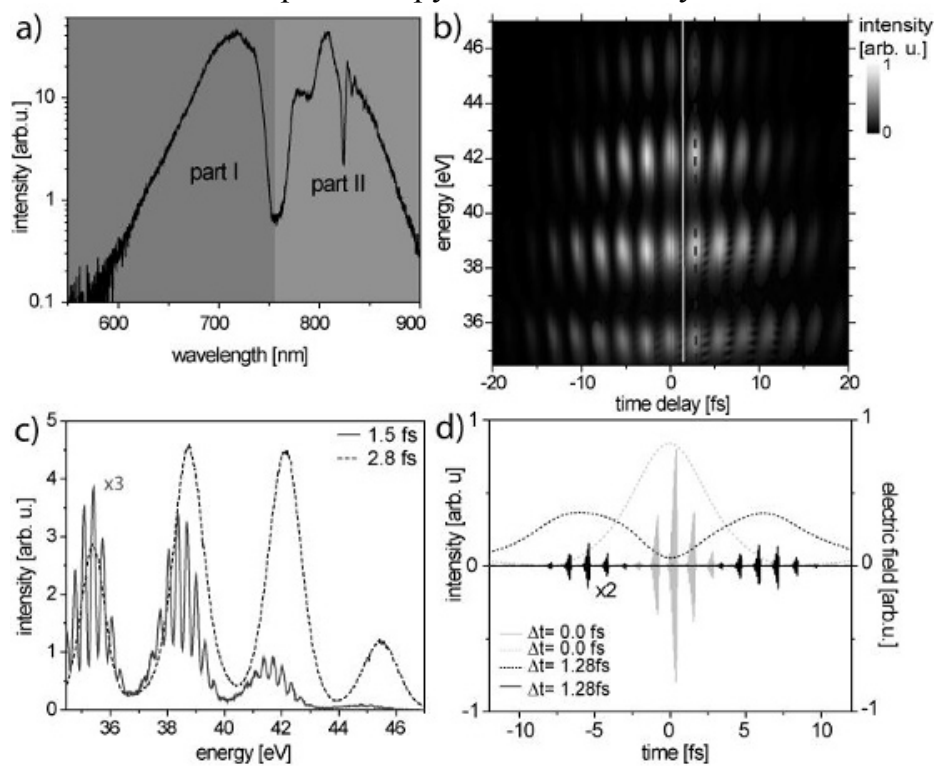

Fig. 2. Spectral effects of split-spectrum HHG control and mechanism of fractional highharmonic comb generation [6]. a) Driver split spectrum with the cut at $755 \mathrm{~nm}$ (symmetric cut, I:II energy ratio 1.53:1). b) Measured time-delay dependent spectrum of high-order harmonics c) Measured regular odd harmonics at 2.8 fs time delay and fractional harmonics at 1.5 fs time delay. d) Simulated driver intensity profiles (dashed lines) and electric fields of attosecond pulse trains (solid lines) at zero and 1.28 fs time delay. The fractional harmonics result from the controlled spectral interference of two attosecond pulse trains when the driving field is shaped such that it exhibits two distinct temporal intensity maxima.

The presented method demonstrates the single-atom-level control of high-harmonics and attosecond pulse trains by utilizing a single broadband shaped laser field. The approach thus represents a step forward on the route towards interferometric control of electron dynamics in atoms and molecules on attosecond time scales.

\section{References}

[1] A. Baltuska et al., "Attosecond control of electronic processes by intense light fields", Nature 421, 611-615 (2003).

[2] E. Mansten et al.,"Spectral Signature of Attosecond Pulse Trains", Phys. Rev. Lett. 102, 083002 (2009).

[3] T. Pfeifer et al., "Controlling the spectral shape of coherent X-rays", Appl. Phys. B 80, 277 (2005).

[4] R. Bartels, S. Backus, E. Zeek, L. Misoguti, G. Vdovin, I. Christov, M. Murnane, and H. Kapteyn, "Shaped-pulse optimization of coherent emission of high-harmonic soft X-rays", Nature 406, 164 (2000).

[5] A. Wirth et al., "Synthesized Light Transients", Science 334, 195-200 (2011).

[6] P. Raith, C. Ott, C. P. Anderson, A. Kaldun, K. Meyer, M. Laux, Y. Zhang, and T. Pfeifer, "Fractional high-order harmonic combs and energy tuning by attosecond-precision split-spectrum pulse control", Appl. Phys. Lett. 100, 121104 (2012).

[7] P. Raith, C. Ott and T. Pfeifer, "Attosecond Twin Pulse Control by Generalized Kinetic Heterodyne Mixing”, Opt. Lett. 36, 283-285 (2011). 